\title{
Modeling of the Division Point of Different Propagation Mechanisms in the Near-Region Within Arched Tunnels
}

\author{
Ke Guan - Zhangdui Zhong · Bo Ai · \\ Cesar Briso-Rodríguez
}

\begin{abstract}
An accurate characterization of the near-region propagation of radio waves inside tunnels is of practical importance for the design and planning of advanced communication systems. However, there has been no consensus yet on the propagation mechanism in this region. Some authors claim that the propagation mechanism follows the free space model, others intend to interpret it by the multi-mode waveguide model. This paper clarifies the situation in the near-region of arched tunnels by analytical modeling of the division point between the two propagation mechanisms. The procedure is based on the combination of the propagation theory and the three-dimensional solid geometry. Three groups of measurements are employed to verify the model in different tunnels at different frequencies. Furthermore, simplified models for the division point in five specific application situations are derived to facilitate the use of the model. The results in this paper could help to deepen the insight into the propagation mechanism within tunnel environments.
\end{abstract}

Keywords Break point - Division point - Modeling · Near-region · Propagation · Tunnel

\section{Introduction}

Propagation modeling in tunnels has always been a complex task in the design of wireless communication systems. Among all cross-sectional shapes, arched tunnels are the most typical ones for modern road, railway and subway systems and therefore it is important to investigate the propagation modeling of this type of tunnels. Two most common categories

\footnotetext{
K. Guan $(\bowtie) \cdot$ Z. Zhong · B. Ai

State Key Laboratory of Rail Traffic Control and Safety, Beijing Jiaotong University, Beijing 100044, China

e-mail: myecone@ hotmail.com

C. Briso-Rodríguez

Escuela Universitaria de Ingeniería Técnica de Telecomunicación, Universidad Politécnica de Madrid, 28031 Madrid, Spain

e-mail: cbriso@diac.upm.es
} 

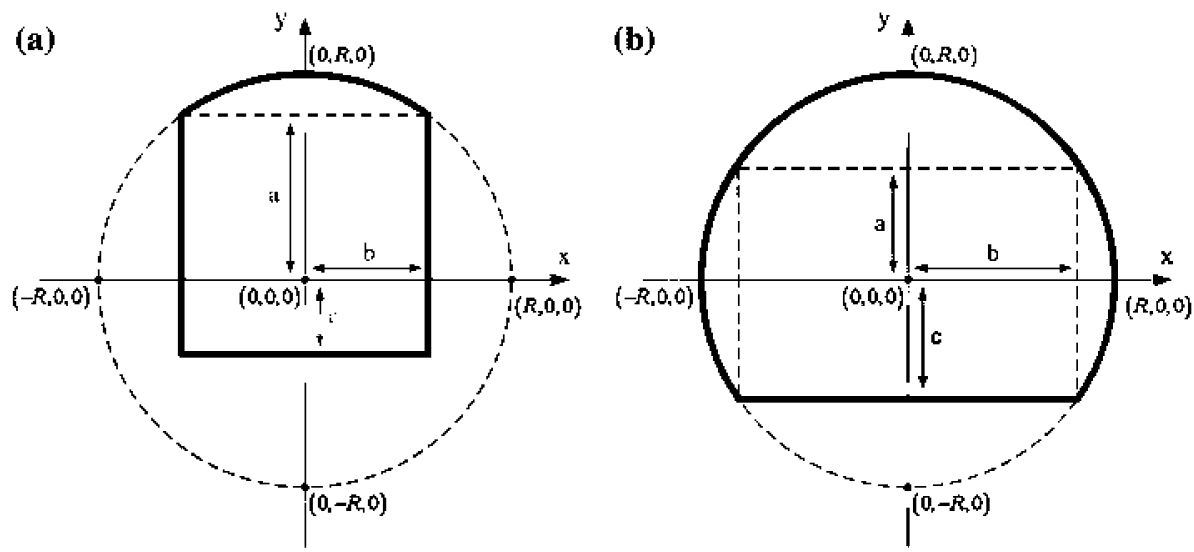

Fig. 1 A cross-section of the arched tunnel "Type I" (a) and "Type II" (b)

of arched tunnels are: Type I,f́6 depicted in Fig. 1a, which consists of flat side walls, flat bottom and arched top, and Type IIf́6 shown in Fig. 1b, which consists of arched side walls, arched top and flat bottom. We have carried out a wide range of propagation measurement campaigns in both types of arched tunnels, in a real subway tunnel as well as in an operational railway tunnel in Spain.

A number of propagation models inside tunnels presented in the last four decades indicate that there is a "critical distance" $[1,2]$, usually called the break point [1-3]. In front of and behind this break point, the propagation characteristics including path loss, shadow fading and small-scale fading are considerably different $[2,4,5]$. Hence, the zone in front of the break point is defined as the near-region; correspondingly, the segment behind it is defined as the far region [6].

The distance from the transmitter to the break point is defined as [1]

$$
Z_{N R}=\operatorname{Max}\left(\frac{W^{2}}{\lambda}, \frac{H^{2}}{\lambda}\right)
$$

where $W$ denotes the width of the rectangular tunnel, $H$ denotes the height of the rectangular tunnel, and $\lambda$ is the signal wavelength in metres, respectively. This equation can be applied in case of arched tunnels, as the electromagnetic field distribution and the attenuation of the modes in arched tunnels are almost the same as in rectangular tunnels [7]. It is worth noting that $Z_{N R}$ is inversely proportional to the wavelength.

The evolution of the wireless communication systems brings us systems with higher and higher operating frequencies. In the dedicated communication field, the typical systems are: TETRA (Terrestrial Trunked Radio) [8], GSM-R (Global System for Mobile Communications for Railway) [9] , CBTC (Communication Based Train Control System) [10], and DSRC (Dedicated Short-range Communications) [11]. Their frequencies are $400 \mathrm{MHz}, 900 \mathrm{MHz}$, 2.4 GHz and 5.8/5.9 GHz, respectively. The similar situation appears in the public communication systems, including GSM (Global System for Mobile Communications) [12], 3G (3rd Generation) [13], Wi-Fi (Wireless Fidelity) [14], and WiMAX (World Interoperability for Microwave Access) [15], having their frequencies rising from $900 / 1800 \mathrm{MHz}, 2 \mathrm{GHz}$ to $2.4 / 5 \mathrm{GHz}$, and $3.8 \mathrm{GHz}$. Apparently, along with the advancement of radio communication systems, the position of the break point within tunnel moves further and further away from 
the transmitter. Therefore, the propagation mechanism in the near-region becomes a major issue.

The propagation characteristics in the far region inside tunnels follow the fundamentalmode waveguide mechanism $[1,2,6]$; however, there has been no consensus on the propagation in the near region within tunnels. Some dissertations are inclined to interpret the propagation in front of the break point with a single ray (free space) theory $[6,16]$, others contend that it should be described by a multi-mode waveguide model $[1,2,17]$. All the cited publications treat the near-region as a whole and interpret the propagation situation by a single mechanism. In fact, there has been much evidence that proves that both propagation mechanisms exist simultaneously. The free space mechanism is established at short ranges and the multi-mode propagation mechanism occurs at longer ranges. Thus, interpreting the propagation in the near-region by merely one mechanism and corresponding model limits the prediction accuracy. To overcome this limitation, a novel structure with a clear division point between different propagation mechanisms is highly desired. The authors of [18] divide the near-region into two segments and model the path loss in each segment. Concerning their division point, the model in the free space is employed to calculate the location [18]. This result has been proven to be reasonable, but it is valid only under certain conditions [19].

In order to clarify the propagation situation in the near-region of tunnels, it is essential to establish a general model for the accurate location of the division point between different mechanisms.

\section{Analytical Modeling of the Propagation Mechanisms and Their Division Point}

\subsection{Geometrical and Electrical Modeling for the Tunnel}

From a theoretical point of view, arched tunnel can be regarded as an equivalent rectangular tunnel. Therefore in this paper, the arched tunnel is treated as an equivalent oversized imperfect hollow rectangular waveguide. The size of the equivalent rectangular waveguide can be computed by taking the main horizontal dimension $W$ close to the tunnel's floor size and computing the vertical dimension $H$ by using the "rule of thumb", i.e.,

$$
H=\sqrt{4 R^{2}-W^{2}}
$$

This idealized geometry is common in modern road and railway tunnels [20].

In order to use the geometrical and modal analysis, the following parameters are required:

- A coordinate system: a three-dimensional Cartesian coordinate system, with its origin located at an angle of the equivalent rectangular tunnel.

- Geometrical dimensions: width of the equivalent rectangular tunnel: $W$, height of the equivalent rectangular tunnel: $H$. The coordinates of the transmitter, receiver and middle point on the line of sight between transmitter and receiver are $P_{t}\left(x_{t}, y_{t}, z_{t}\right), P_{r}\left(x_{r}, y_{r}, z_{r}\right)$ and $P_{0}\left(x_{0}, y_{0}, z_{0}\right)$; their relations are expressed by

$$
x_{0}=\frac{x_{r}+x_{t}}{2}, y_{0}=\frac{y_{r}+y_{t}}{2}, z_{0}=\frac{z_{r}+z_{t}}{2}
$$

- Electrical parameters: relative permittivity for vertical and horizontal walls: $\varepsilon_{v}$ and $\varepsilon_{h}$, with the typical values for concrete: $\varepsilon_{v}=\varepsilon_{h}=5$ [1] 


\subsubsection{Propagation Loss in the Free Space Propagation Segment}

In the adjacent region of the transmitter antenna, the angles of incidence from the ray to the wall (vertical and horizontal) are high, resulting in high attenuation of reflected rays, whereas the path difference between direct and reflected rays may also cause additional attenuation. Thus, only the direct ray significantly contributes to the strength of the received signal. The channel loss in this segment follows the free space loss attenuation [21]

$$
P L(d B)=-10 \log _{10}\left[\frac{\lambda^{2}}{(4 \pi)^{2}\left|z_{r}-z_{t}\right|^{2}}\right]
$$

where $\left|z_{r}-z_{t}\right|$ is the distance between transmitter and receiver in metres, and $\lambda$ is the signal wavelength.

\subsubsection{Propagation Loss in the Multi-Mode Waveguide Segment}

According to the modal theory, a rectangular tunnel can be regarded as an oversized imperfect hollow rectangular waveguide. Since the UHF is considerably higher than the cutoff frequency of the fundamental modes, a wide range of $E_{m n}$ multiple modes propagate inside tunnels when the free space segment ends [18]. Using modal theory, the losses of horizontally and vertically polarized $E_{m n}$ modes can be given by

$$
\begin{aligned}
& \alpha(m, n)^{h}=4.343 \lambda^{2}\left(\frac{m^{2} \varepsilon_{v}}{W^{3} \sqrt{\varepsilon_{v}-1}}+\frac{n^{2}}{H^{3} \sqrt{\varepsilon_{h}-1}}\right) d B / m \\
& \alpha(m, n)^{v}=4.343 \lambda^{2}\left(\frac{m^{2}}{W^{3} \sqrt{\varepsilon_{v}-1}}+\frac{n^{2} \varepsilon_{h}}{H^{3} \sqrt{\varepsilon_{h}-1}}\right) d B / m
\end{aligned}
$$

With the propagation constants offered above, the propagation loss in the multi-mode waveguide segment can be calculated by considering the modes for both polarizations

$$
L_{m n}^{v / h}(d B)=10 \lg \left[\sum_{i=1}^{m} \sum_{j=1}^{n} \times \sqrt{10^{2 \alpha(i, j)^{h}}\left|z_{r}-z_{t}\right|+10^{2 \alpha(i, j)^{v}\left|z_{r}-z_{t}\right|}}\right]
$$

\subsection{Division Point Between Different Propagation Mechanisms}

\subsubsection{The Core Idea of Localization of the Division Point}

In optics and radio communications, Fresnel zones are elliptical in shape with the transmitter and receiver antenna at their foci. The innermost ellipsoid is called the first Fresnel zone, which contains the strongest radio signal.

Along with the increase of the distance between receiver and transmitter, the first Fresnel zone becomes larger and larger. Hence, the propagation process can be described as follows.

Firstly, when the receiver stays in the adjacent region of the transmitter, the first Fresnel zone is not large enough to touch any wall of the tunnel. In this case the volume contained within the first Fresnel zone is not blocked by any obstruction, so the diffraction effects may be neglected. Meanwhile, the grazing angles of incidence from the ray to the wall are high leading to high attenuation of reflected rays. Furthermore, the path difference between the 
direct ray and the rays reflecting off those objects out of the first Fresnel zone may cause additional attenuation. All these render the already weak reflected rays even weaker. As a consequence, only the direct ray significantly contributes to the strength of the received signal. Thus, the free space loss channel model can be applied in this region.

Subsequently, with the receiver moving further away, the first Fresnel zone will touch the wall at one point, then the reflection starts to occur inside the first Fresnel zone and effectively reinforces the signal strength at the receiver. This means the end of the free space propagation mechanism.

Further from that point, the first Fresnel zone becomes unclear along with its own expansion. The effect can be treated as the process in which the walls of the tunnel penetrate the first Fresnel zone and generate many effective reflected rays, which are regarded as modes in waveguide theory. Hence, the multi-mode waveguide propagation mechanism starts.

Based on the above analysis, it is manifest that the point where the first Fresnel zone is tangent to the walls of the tunnel is the division point between the two propagation mechanisms. However, locating it is definitely not an easy task. Since the interaction between the first Fresnel zone and the walls depends on a great number of factors, such as the locations of the transmitting antenna and receiving antenna, the dimensions of the tunnel, the operating frequency, etc., the computational time would be intolerable if all the elements were considered when we track the interaction and its change law. Hence, in order to accelerate the process, it is desirable to find a simple parameter representing the interaction.

According to geometry, it is easy to determine the distance between the tangent line/curve (of the Maximum Fresnel zone plate and the walls) and the middle point (of the line of sight between transmitter and receiver). If this distance is larger than the radius of the Maximum first Fresnel zone plate, the first Fresnel zone can be treated as almost clear. It is true that in this case some parts inside the first Fresnel zone could still be blocked. But since the first Fresnel zone is a flat ellipsoid, such kind of slight obstruction does not lead to many effective reflected rays or obvious diffractive loss. Hence, the free space propagation model can still work. When this distance is smaller than the radius, that means even the widest part of the first Fresnel zone is blocked, more severe obstruction occurs in the other parts. Thus, the relative relation of this distance and the radius can be employed to reflect the interaction between the first Fresnel zone and the walls to some extent. Furthermore, the location of the division point can be deduced when the distance and the radius are equal.

\subsubsection{Two Types of Arched Tunnels}

To grasp the accurate propagation mechanism situation, it is essential to model the location of the division point between the free space propagation segment and the multi-mode waveguide segment. Figure $1 \mathrm{a}$ and $\mathbf{b}$ demonstrate the cross-sectional geometry for both types of arched tunnels. It is noteworthy that both arched tunnel "Type I" and "Type II" can be seen as a combination of a circular and a rectangular tunnel, but in different configurations. Hence, the division point can be modeled in circular tunnel and rectangular tunnel independently, then determined by their specific combinations.

\subsubsection{Geometrical Analysis in the Case of Circular Tunnel}

The tunnel is analyzed by being regarded as a circular tunnel. Figure 2 depicts the threedimensional geometry schematic diagram of a circular tunnel, transmitter, receiver, line of sight, and the Maximum first Fresnel zone. According to the three-dimensional solid 


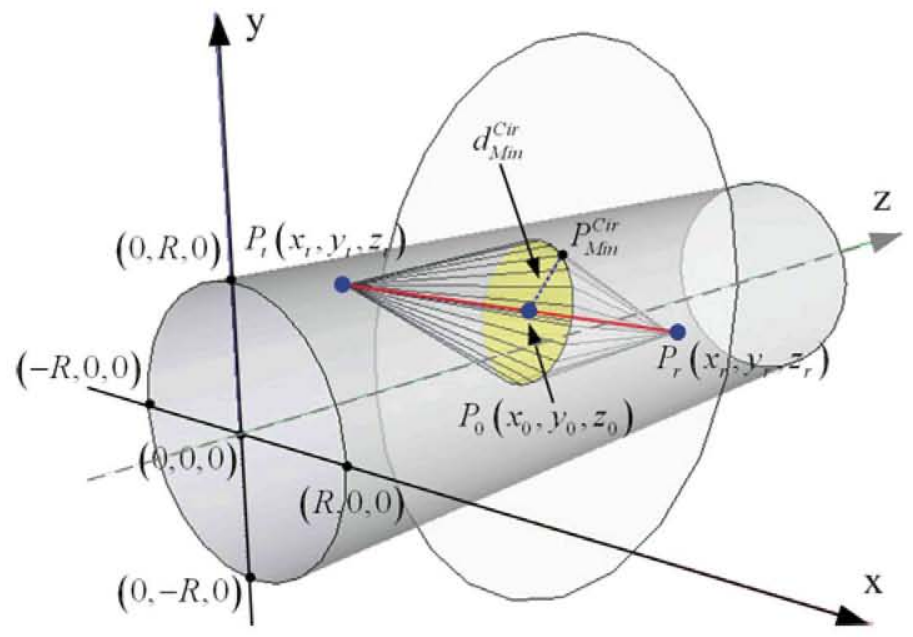

Fig. 2 Detailed schematic diagram of the propagation inside circular tunnels with the first Fresnel zone clearance

geometry, the surface of the circular tunnel is the set of points, the coordinates of which $x, y$ satisfy the following equation

$$
x^{2}+y^{2}=R^{2}
$$

with z-coordinates completely arbitrary. The Maximum Fresnel zone plate can be expressed by a plane of a general type

$$
\begin{aligned}
& \left(x_{r}-x_{t}\right)\left(x-\frac{x_{r}+x_{t}}{2}\right)+\left(y_{r}-y_{t}\right)\left(y-\frac{y_{r}+y_{t}}{2}\right) \\
& +\left(z_{r}-z_{t}\right)\left(z-\frac{z_{r}+z_{t}}{2}\right)=0
\end{aligned}
$$

The intersection between the Maximum Fresnel zone plane and the surface of circular tunnel is a curve which can be defined as

$$
\left\{\begin{array}{c}
\left(x_{r}-x_{t}\right)\left(x-\frac{x_{r}+x_{t}}{2}\right)+\left(y_{r}-y_{t}\right)\left(y-\frac{y_{r}+y_{t}}{2}\right) \\
+\left(z_{r}-z_{t}\right)\left(z-\frac{z_{r}+z_{t}}{2}\right)=0 \\
x^{2}+y^{2}=R^{2}
\end{array}\right.
$$

If we define the first equation as a function $f(x, y, z)$ as

$$
\begin{aligned}
f(x, y, z)= & \left(x_{r}-x_{t}\right)\left(x-\frac{x_{r}+x_{t}}{2}\right)+\left(y_{r}-y_{t}\right)\left(y-\frac{y_{r}+y_{t}}{2}\right) \\
& +\left(z_{r}-z_{t}\right)\left(z-\frac{z_{r}+z_{t}}{2}\right)
\end{aligned}
$$

and the second equation as a function $g(x, y, z)$ as

$$
g(x, y, z)=x^{2}+y^{2}-R^{2}
$$

in order to find the minimal distance between the intersection (curve) and the middle point $P_{0}\left(x_{0}, y_{0}, z_{0}\right)$ on the line of sight, the Lagrange multiplier method and seeking the extremum are employed. Let us construct a function as follows: 


$$
\begin{gathered}
F(x, y, z, \xi, \mu)=\left(x-\frac{x_{r}+x_{t}}{2}\right)^{2}+\left(y-\frac{y_{r}+y_{t}}{2}\right)^{2} \\
+\left(z-\frac{z_{r}+z_{t}}{2}\right)^{2}+\xi \cdot f(x, y, z)+\mu \cdot g(x, y, z)
\end{gathered}
$$

where $\xi, \mu$ are the Lagrange multipliers. By seeking a partial derivative of $\mathrm{x}, \mathrm{y}$ and $\mathrm{z}$, respectively, Eq. 13 can be transformed as follows:

$$
\begin{aligned}
\frac{\partial F}{\partial x}= & 2\left(x-\frac{x_{r}+x_{t}}{2}\right)+\xi \cdot\left(x_{r}-x_{t}\right)+\mu \cdot 2 x=0 \\
\frac{\partial F}{\partial y}= & 2\left(y-\frac{y_{r}+y_{t}}{2}\right)+\xi \cdot\left(y_{r}-y_{t}\right)+\mu \cdot 2 y=0 \\
\frac{\partial F}{\partial z}= & 2\left(z-\frac{z_{r}+z_{t}}{2}\right)+\xi\left(z_{r}-z_{t}\right)=0 \\
\frac{\partial F}{\partial \xi}= & \left(x_{r}-x_{t}\right)\left(x-\frac{x_{r}+x_{t}}{2}\right)+\left(y_{r}-y_{t}\right)\left(y-\frac{y_{r}+y_{t}}{2}\right) \\
& +\left(z_{r}-z_{t}\right)\left(z-\frac{z_{r}+z_{t}}{2}\right)=0 \\
\frac{\partial F}{\partial \mu}= & x^{2}+y^{2}-R^{2}=0
\end{aligned}
$$

By seeking the simultaneous solution of (14)-(18), the coordinate of intersection point with the minimal distance to $P_{0}\left(x_{0}, y_{0}, z_{0}\right)$ can be obtained: $p_{\text {Min }}^{\mathrm{Cir}}\left(x^{\mathrm{Cir}}\left(z_{r}\right), y^{\mathrm{Cir}}\left(z_{r}\right), z^{\mathrm{Cir}}\left(z_{r}\right)\right)$. Therefore, the minimal distance between $P_{0}$ and the intersection (curve) between the Maximum Fresnel zone plane and the wall of circular tunnel can be expressed as

$$
\begin{aligned}
d_{\text {Min }}^{C i r}\left(z_{r}\right)= & {\left[\left(x^{C i r}\left(z_{r}\right)-\frac{x_{r}+x_{t}}{2}\right)^{2}+\left(y^{C i r}\left(z_{r}\right)-\frac{y_{r}+y_{t}}{2}\right)^{2}\right.} \\
& \left.+\left(y^{C i r}\left(z_{r}\right)-\frac{y_{r}+y_{t}}{2}\right)^{2}+\left(z^{C i r}\left(z_{r}\right)-\frac{z_{r}+z_{t}}{2}\right)^{2}\right]^{\frac{1}{2}}
\end{aligned}
$$

\subsubsection{Geometrical Analysis in the Case of Rectangular Tunnel}

As shown in Fig. 3, the tunnel is treated as a rectangular tunnel. The process is similar to the circular tunnel but easier, since the wall of the rectangular tunnel is not a curved surface but a plane surface, which can be expressed as follows:

$$
A x+B y+C z+D=0
$$

The tangent line is expressed by the simultaneous Eqs. 9 and 20. Similarly, define (9) and (20) as function $f(x, y, z)$ and $g(x, y, z)$, respectively. Using the Lagrange multiplier method, we construct the objective function as follows:

$$
\begin{gathered}
G(x, y, z, \varphi, \psi)=\left(x-\frac{x_{r}+x_{t}}{2}\right)^{2}+\left(y-\frac{y_{r}+y_{t}}{2}\right)^{2} \\
+\left(z-\frac{z_{r}+z_{t}}{2}\right)^{2}+\varphi \cdot f(x, y, z)+\psi \cdot h(x, y, z)
\end{gathered}
$$




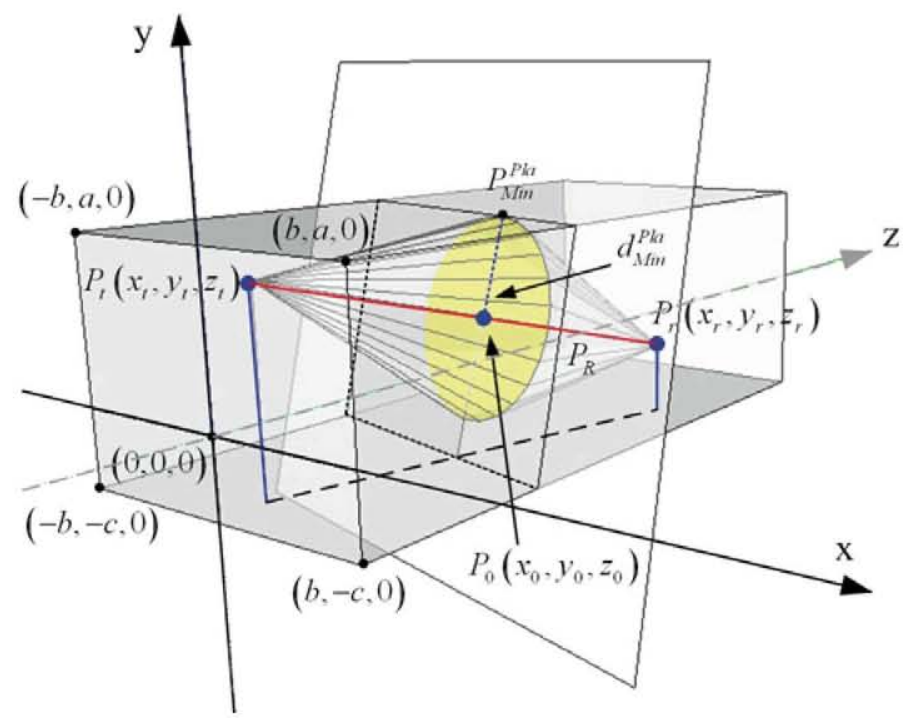

Fig. 3 Detailed schematic diagram of the propagation inside rectangular tunnels with the first Fresnel zone clearance

where $\psi$ is the Lagrange multiplier. By seeking partial derivative of $\mathrm{x}, \mathrm{y}$ and $\mathrm{z}$, respectively, Eq. 21 can be transformed to

$$
\begin{aligned}
\frac{\partial G}{\partial x}= & 2\left(x-\frac{x_{r}+x_{t}}{2}\right)+\varphi \cdot\left(x_{r}-x_{t}\right)+\psi \cdot A x=0 \\
\frac{\partial G}{\partial y}= & 2\left(y-\frac{y_{r}+y_{t}}{2}\right)+\varphi \cdot\left(y_{r}-y_{t}\right)+\psi \cdot B y=0 \\
\frac{\partial G}{\partial z}= & 2\left(z-\frac{z_{r}+z_{t}}{2}\right)+\varphi \cdot\left(z_{r}-z_{t}\right)+\psi \cdot C z=0 \\
\frac{\partial G}{\partial \varphi}= & \left(x_{r}-x_{t}\right)\left(x-\frac{x_{r}+x_{t}}{2}\right)+\left(y_{r}-y_{t}\right)\left(y-\frac{y_{r}+y_{t}}{2}\right) \\
& +\left(z_{r}-z_{t}\right)\left(z-\frac{z_{r}+z_{t}}{2}\right)=0 \\
\frac{\partial G}{\partial \psi}= & A x+B y+C z+D=0
\end{aligned}
$$

By solving (22)-(26), the location of the intersection point with the minimal distance to $P_{0}$ can be obtained: $p_{\text {Min }}^{P l a}\left(x^{P l a}\left(z_{r}\right), y^{P l a}\left(z_{r}\right), z^{P l a}\left(z_{r}\right)\right)$. Then, the minimal distance between $P_{0}$ and the intersection (line) are derived as:

$$
\begin{aligned}
d_{\text {Min }}^{\text {Pla }}\left(z_{r}\right)= & {\left[\left(x^{\text {Pla }}\left(z_{r}\right)-\frac{x_{r}+x_{t}}{2}\right)^{2}+\left(y^{\text {Pla }}\left(z_{r}\right)-\frac{y_{r}+y_{t}}{2}\right)^{2}\right.} \\
& \left.+\left(z^{\text {Pla }}\left(z_{r}\right)-\frac{z_{r}+z_{t}}{2}\right)^{2}\right]^{\frac{1}{2}}
\end{aligned}
$$


After deducing the Algorithm of $d_{M i n}^{C i r}\left(z_{r}\right)$ and $d_{M i n}^{P l a}\left(z_{r}\right)$ from circular and rectangular tunnels, respectively, we need to determine the minimal distance $d_{M i n}\left(z_{r}\right)$ in the arched tunnel case according to the two types of combinations.

Based on the propagation theory, the radius of the first Fresnel zone is determined by

$$
r_{1}=\sqrt{\frac{\lambda d_{1} d_{2}}{d_{1}+d_{2}}}
$$

where $d_{1}$ denotes the distance between the transmitter and the point of interaction between the line of sight and the first Fresnel zone, $d_{2}$ denotes the distance between the receiver and the point of interaction. When the point of interaction is the middle point $P_{0}$, then $d_{1}=d_{P_{t} P_{0}}=d_{2}=d_{P_{0} P_{r}}=\frac{1}{2} d_{P_{t} P_{r}}$. At this point, the radius gets the maximum value of the first Fresnel zone plate

$$
r_{1 M a x}\left(z_{r}\right)=\frac{1}{2} \sqrt{\lambda d_{P_{t} P_{r}}}
$$

According to the propagation theory, the free space loss channel model can be applied if the first Fresnel zone is free of any obstacles. Therefore, the division point between two propagation mechanisms locates at $z_{r}$ which is the minimal positive real root of the following equation

$$
r_{1 \operatorname{Max}}\left(z_{r}\right)=d_{\operatorname{Min}}\left(z_{r}\right)
$$

This means that the Maximum first Fresnel zone plate first touches the surface of arched tunnels; even the widest part of the first Fresnel zone starts to be blocked from this point onwards.

In Fig. 4 the propagation inside the arched tunnel "Type I" with the first Fresnel zone clearance is shown. In "Type I", two vertical walls and the floor in the rectangular tunnel as well as the arched roof in the circular tunnel can possibly obstruct the first Fresnel zone, thus substituting the roof function to (8) and the wall/floor function to (20):

- Left vertical side wall: $x=-b$;

- Right vertical side wall: $x=b$;

- Bottom/Floor: $y=-c$;

- Arched top/roof: $x^{2}+y^{2}=R^{2},|x| \leq b, a \leq y \leq R$;

$d_{\text {Min }}^{\text {Cir-R }}\left(z_{r}\right), d_{\text {Min }}^{\text {Pla-R }}\left(z_{r}\right), d_{\text {Min }}^{\text {Pla-L }}\left(z_{r}\right)$ and $d_{\text {Min }}^{\text {Pla-F }}\left(z_{r}\right)$ corresponding to the minimal distance from $P_{0}$ to the intersection (line/curve) on the arched roof, the right/left wall and the floor can be obtained. By using (30), the division point location of $z_{r_{M i n}}^{C i r-R}, z_{r_{M i n}}^{\text {Pla }}{ }_{\text {Min }}, z_{r_{\text {Min }}}^{\text {Pla-L }}$ and $z_{r_{M i n}}{ }_{\text {Pa }}$ corresponding to the touching of the Maximum first Fresnel zone and the arched roof, right wall, left wall and floor of arched tunnels, respectively, can be derived as:

$$
\begin{aligned}
& z_{r_{\text {Min }}^{C i r-R}}^{\text {Cin }}=\operatorname{Min}\left\{z_{r} \mid r_{1 \text { Max }}\left(z_{r}\right)=d_{\text {Min }}^{\text {Cir-R }}, z_{r} \in R^{+}\right\} \\
& z_{r_{\text {Min }}^{\text {Pla-R }}}=\operatorname{Min}\left\{z_{r} \mid r_{1 \text { Max }}\left(z_{r}\right)=d_{\text {Min }}^{\text {Pla-R }}, z_{r} \in R^{+}\right\} \\
& z_{r_{\text {Min }}^{\text {Pla }}}^{\text {Pla }}=\operatorname{Min}\left\{z_{r} \mid r_{1 \text { Max }}\left(z_{r}\right)=d_{\text {Min }}^{\text {Pla-L }}, z_{r} \in R^{+}\right\} \\
& z_{r_{\text {Min }}^{\text {Pla-F }}}^{\text {Pla }}=\operatorname{Min}\left\{z_{r} \mid r_{1 \text { Max }}\left(z_{r}\right)=d_{\text {Min }}^{\text {Pla-F }}, z_{r} \in R^{+}\right\}
\end{aligned}
$$




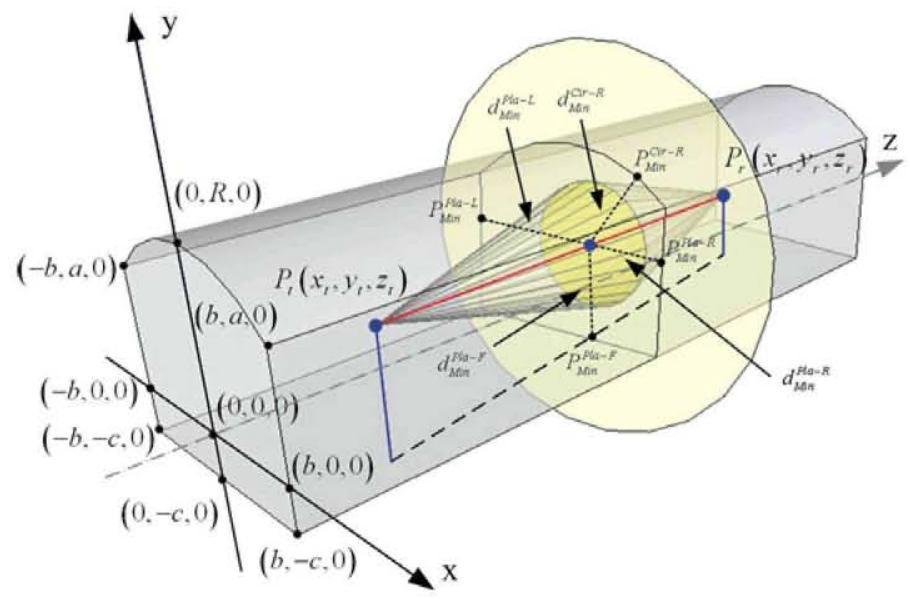

Fig. 4 Detailed schematic diagram of the propagation inside the arched tunnel "Type I" with the first Fresnel zone clearance

Then, the division point between the two propagation mechanisms inside the arched tunnel "Type I" is located at $z_{r}$ :

$$
z_{r}=\operatorname{Min}\left(z_{\text {Min }}^{\text {Cir-R }}, z_{r_{\text {Min }}}^{P l a-R}, z_{r_{\text {Min }}}^{\text {Pla-L }}, z_{r_{\text {Min }}}^{\text {Pla-F }}\right)
$$

Figure 5 illustrates the propagation of the clearance of the first Fresnel zone inside arched tunnel "Type II". In "Type II", only the floor in the rectangular tunnel and the arched roof/wall in the circular tunnel can possibly obstruct the first Fresnel zone, thus substituting the arched roof's functions to (8) and the floor's function to (20):

- Floor: $y=-c$;

- Arched roof: $x^{2}+y^{2}=R^{2},-c \leq y \leq R$;

$d_{\text {Min }}^{\text {Cir }-R / W}\left(z_{r}\right)$ and $d_{\text {Min }}^{\text {Pla-F }}\left(z_{r}\right)$ can be derived. By employing (30), the division point location of $z_{r_{\text {Min }}} \mathrm{Cir}^{-R}$ and $z_{r_{\text {Min }}}$ Pla-F corresponding to the touching of the Maximum first Fresnel zone and the arched roof/floor and the floor of tunnels, respectively, can be obtained:

$$
\begin{aligned}
& z_{r_{\text {Min }}}^{\operatorname{Cir}-R / W}=\operatorname{Min}\left\{z_{r} \mid r_{1 \text { Max }}\left(z_{r}\right)=d_{\text {Min }}^{\text {Cir-R }}, z_{r} \in R^{+}\right\} \\
& z_{r_{\text {Min }}}^{\text {Pla-F }}=\operatorname{Min}\left\{z_{r} \mid r_{1 \text { Max }}\left(z_{r}\right)=d_{\text {Min }}^{\text {Pla-F }}, z_{r} \in R^{+}\right\}
\end{aligned}
$$

Then, the division point inside the arched tunnel "Type II" is located at $z_{r}$ :

$$
z_{r}=\operatorname{Min}\left(z_{r_{M i n}}^{\operatorname{Cir}-R / W}, z_{r} \stackrel{\text { Min }}{\operatorname{Min}-F}\right)
$$

\section{Division Point Model Validation}

Three groups of measurement campaigns previously published have been used for validating the model of the division point presented in the previous section. The chosen tunnels possess great diversity: the tunnel type includes road tunnel, pedestrian tunnel and railway tunnel; 


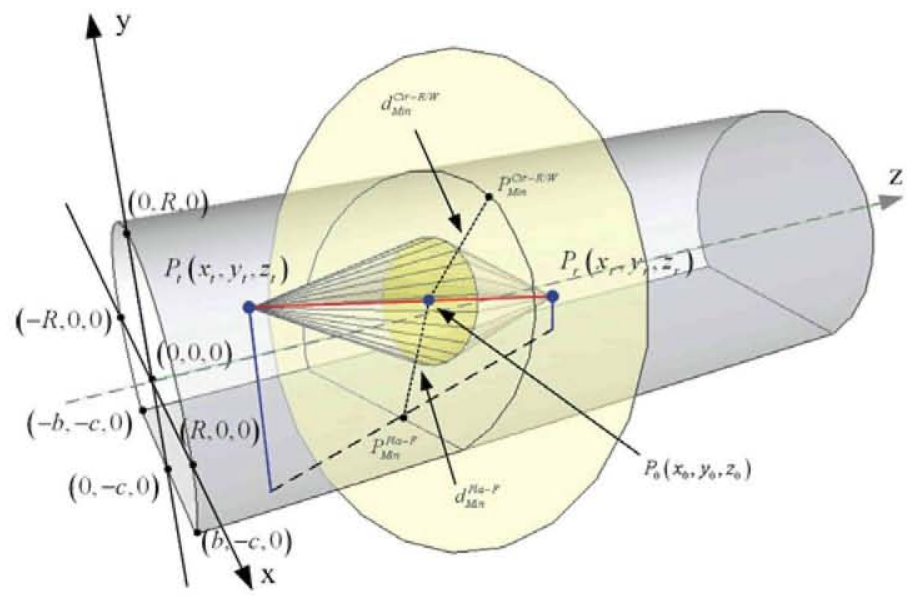

Fig. 5 Detailed schematic diagram of the propagation inside the arched tunnel "Type II" with the first Fresnel zone clearance

the operating frequency involves 400 and $900 \mathrm{MHz}$. Relevant details and parameters in these measurements are cited as follows:

- The first data set consists of the measurements taken in one of the longest tunnels of the new 450-km high-speed train line connecting Madrid and Lleida in Spain [1]: $\lambda=0.33, R=6.2, x_{t}=6, y_{t}=0, z_{t}=0, x_{r}=3, y_{r}=0, A=0, B=1, C=$ $0, D=3$. By seeking the simultaneous solution of (14)-(18), the coordinate of intersection point with the minimal distance to $P_{0}\left(x_{0}, y_{0}, z_{0}\right)$ in the circular tunnel can be obtained: $p_{\text {Min }}^{\text {Cir }-R / W}(6.2,0,17.57)$. By substituting relevant parameter into (9) and (20), the minimal positive real root of the simultaneous solutions of (22)-(26) is the coordinate of intersection point with the minimal distance to $P_{0}\left(x_{0}, y_{0}, z_{0}\right)$ in the rectangular tunnel: $p_{\text {Min }}^{P l a-F}(4.5,-3,54.03)$. By solving (36) and (37), $z_{r_{M i n}}^{\mathrm{Cir}-R / W}=34.84, z_{r_{M i n}}^{P l a-F}=$ 108.07. Hence, according to (38), the division point locates at $z_{r}=34.84$ in this case.

- The second data set of received signal strength consists of the measurements performed in a railway tunnel typical for Europe at $400 \mathrm{MHz}$. The tunnel is $520 \mathrm{~m}$ long and originally engineered for a railway, but the line was closed and it is now being used by pedestrians and cyclists [18]: $\lambda=0.75, R=2.35, x_{t}=0, y_{t}=0, z_{t}=0, x_{r}=0, y_{r}=0, A=0, B=$ $1, C=0, D=1.5$. By jointly solving (14)-(18), we can get: $p_{\text {Min }}^{\mathrm{Cir}-R / W}(0,2.35,13.48)$. By simultaneously solving (25)-(29), we can obtain: $p_{\text {Min }}^{\text {Pla-F }}(0,-1.5,6.85)$. By solving (36) and (37), $z_{r_{\text {Min }}}^{\mathrm{Cir}-R / W}=27, z_{r_{\text {Min }}}^{\text {Pla-F }}=13.65$. Then using (38), the division point is located at $z_{r}=13.65$.

- The third set of measurements are carried out in a dual carriageway road tunnel connecting Austria and Slovenia at $400 \mathrm{MHz}$. The tunnel was closed in one direction at the time, while the second lane was in normal operation [18]: $\lambda=0.75, R=5.28, x_{t}=3.2, y_{t}=$ $-0.8, z_{t}=0, x_{r}=3.2, y_{r}=-0.8, A=0, B=1, C=0, D=2.5$. By inserting the relevant parameters into (8), (9) and (20), and then applying the model, we can obtain: $z_{r_{\text {Min }}}^{\mathrm{Cir}-R / W}=20.94, z_{r_{\text {Min }}}^{\text {Pla-F }}=15.41$. Equation 38 indicates that the division point is located at $z_{r}=15.41$, which means that the Maximum first Fresnel zone plate first touches the roof of the arched tunnel. 
Table 1 Comparison of the division point results for different types of tunnels between the model and the measurements

\begin{tabular}{llll}
\hline Tunnel & $\begin{array}{l}\text { Frequency } \\
(\mathrm{GHz})\end{array}$ & $\begin{array}{l}\text { Measured } \\
\text { result }(\mathrm{m})\end{array}$ & $\begin{array}{l}\text { Theoretical } \\
\text { prediction (m) }\end{array}$ \\
\hline $\begin{array}{l}\text { Railway tunnel in } \\
\quad \begin{array}{l}\text { Spain [1] } \\
\text { Pedestrian tunnel in } \\
\quad \text { Europe [18] } \\
\text { Road tunnel } \\
\text { Austria-Slovenia }\end{array}\end{array}$ & 0.9 & 35 & 34.84 \\
{$[18]$} & 0.4 & 15 & 13.65 \\
\hline
\end{tabular}

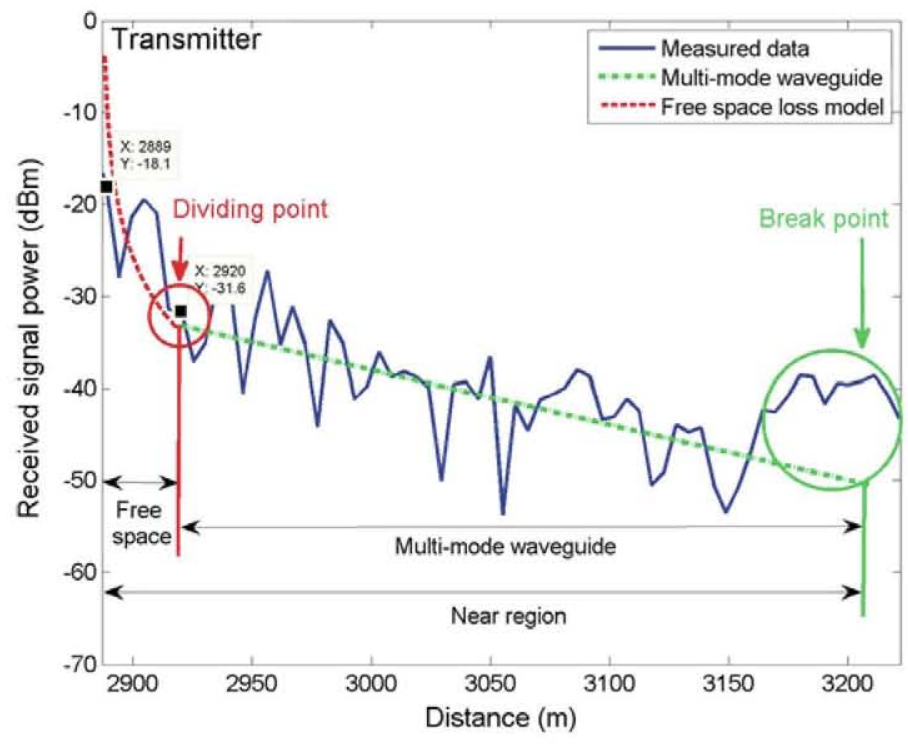

Fig. 6 Comparison between measured and theoretical results on the propagation mechanisms and their division points in the near-region within arched tunnels

Table 1 illustrates the comparison of the division point inside tunnels between the model and the measurements. The location of the division point is extracted from the measurements in this way: the free space propagation model was compared with the measured received signal power, then the point, in front of which the fitting is good and behind which is bad, was found. The theoretical location of the division point is predicted by the model proposed in this paper. As shown in Table 1, the results from the comparison indicate that the model for the division point is performing well in different types of tunnels at various frequencies.

To depict the effect of the entire propagation model in the near-region of arched tunnels, the results of the measurements carried out in a railway tunnel in Spain at $900 \mathrm{MHz}$ [1] have been employed. As shown in Fig. 6, the division point clearly separates the two propagation regions. The propagation in the segment in front of the division point follows the free space mechanism; the signal strength in this part drops like in the free space and is completely independent of the propagation environment. The segment behind the division point is dominated by multi-mode waveguide mechanism and the multi-mode waveguide loss model applied here is in good agreement with the measured data. 

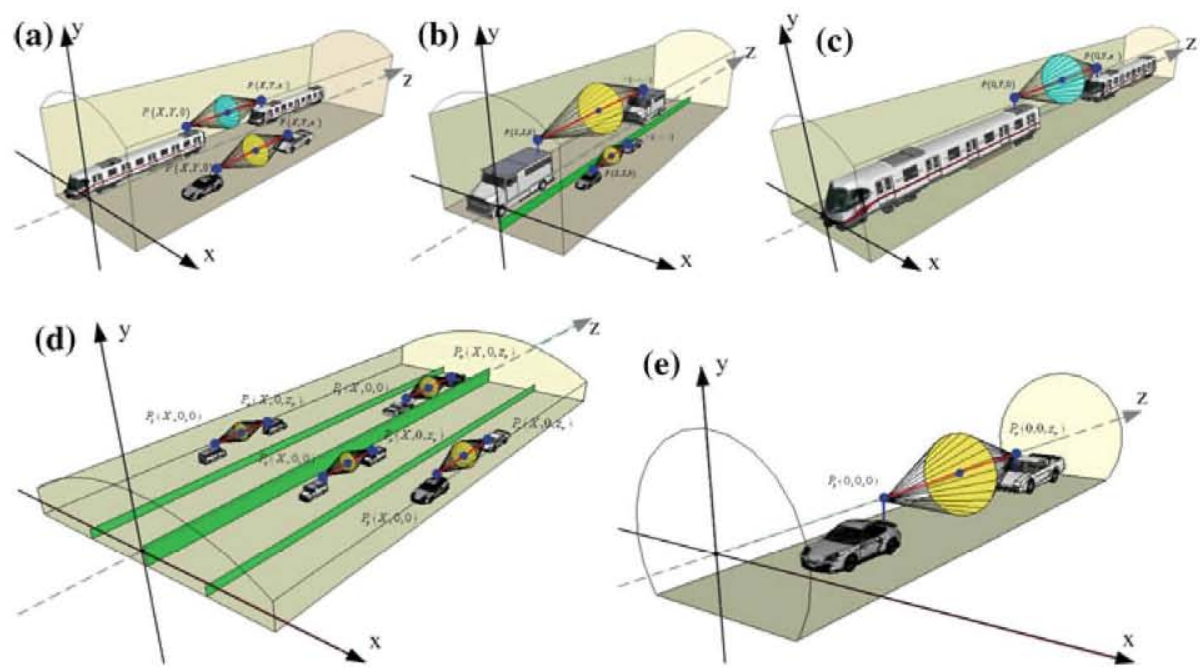

Fig. 7 a Special situation one; b special situation two; c special situation three; $\mathbf{d}$ special situation four; e special situation five

All the validation results and comparison offered above indicate that the analytical model for the propagation mechanisms and their division point in the near-region within arched tunnels presented in this paper is valid and easy to use.

\section{Division Point Model Simplification and Discussion}

In some applications, the locations of transmitting and receiving antennas, as well as the motion trajectories of mobile stations follow certain rules. In this section, the simplified formulas of the diving point model are deduced corresponding to five application situations.

Figure 7a shows Special Situation One: in some systems, such as Dedicated Short Range Communications (DSRC) [11], the communication is going on between different vehicles (car or carriage). In this case, the transmitter and the receiver always have similar heights and similar tracks: $x_{t}=x_{r}=X, y_{t}=y_{r}=Y$. Figure 7b depicts Special Situation Two, which can be met in DSRC, particularly in the dual carriageway road tunnel where the antennas of transmitter and receiver on-vehicle have similar horizontal and vertical distance from the center of the cross-section: $x_{t}=x_{r}=y_{t}=y_{r}=L$. Figure 7c illustrates Special Situation Three: unlike Special Situation Two, this situation usually occurs in the one-way narrow tunnel, for both car and train. That means all the communication units move along the central track with similar high antennas: $x_{t}=x_{r}=0, y_{t}=y_{r}=Y$. Figure $7 \mathrm{~d}$ demonstrates Special Situation Four: like Special Situation Three, this situation requirements can be met in multi-way wide tunnel, for both cars and trains. In this case, all the communication units move along the same track and the antennas' heights approximately equal the center of the cross-section: $x_{t}=x_{r}=X, y_{t}=y_{r}=0$; Fig. 7e shows Special Situation Five: in some long tunnels, especially with the operating frequency of several $\mathrm{GHz}$, the near-region is very long. In this case, by using the modal theory, the transmitter and the receiver can be approximated to be located at the center of the tunnel's cross-section: $x_{t}=x_{r}=0$, $y_{t}=y_{r}=0$. 
Table 2 Simplification of the division point model in certain special situations

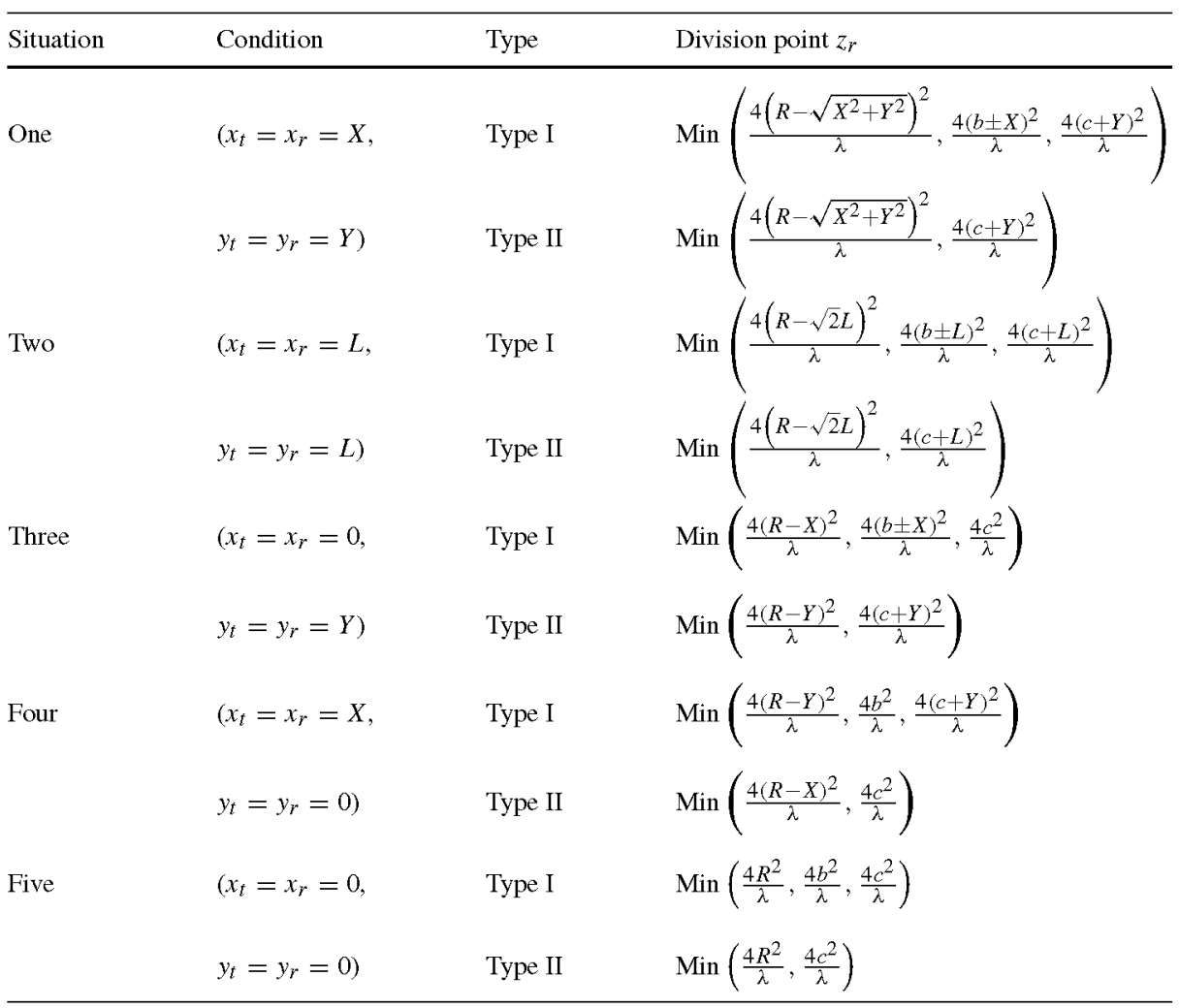

By employing the model presented in this paper, the location of the division point can be expressed by simple formulas corresponding to both "Type I" and "Type II", in each special case. Details are shown in Table 2.

All the simplified formulas provide an easy way to determine the location of the areas corresponding to different propagation mechanisms in the near-region, under the realistic application scenarios. Summarizing the general character of the simplified formulas, we have found that the minimal absolute distance between antennas and any of the tunnel surfaces is the dominant factor in the calculation of these cases. This conclusion can be very useful for the system designer to control different mechanism-based propagation areas in the near-region within tunnels.

\section{Conclusion}

This paper clarifies the propagation situation in the near-region of arched tunnels. The main contribution of this work is the presentation of a general analytical approach and a model for localization of the division point between the free space propagation mechanism and the multi-mode waveguide propagation mechanism. The core idea of this procedure is to use a specified distance to track the interaction of the first Fresnel zone and the walls of 
tunnel. Three groups of measurements have proved that the model is valid in different types of tunnels at different operating frequencies.

Simplified formulas allow the model to be easily applied in many realistic situations. It has been found that in these cases the minimal absolute distance between the antennas and any of the tunnel surfaces dominates the determination of the location of the division point. This conclusion can effectively help system designers to control different mechanism-based propagation areas.

The analysis, approach, and model in this paper can be essential for deeper understanding of the propagation mechanism inside tunnels, and can be used in the realistic radio system design for link and system level simulations.

Acknowledgments We would like to thank the following for their support: the NNSF of China under Grant 60830001, Program for New Century Excellent Talents in University under Grant NCET-09-0206, Beijing NSF 4112048, the Key Project of State Key Lab. of Rail Traffic Control and Safety under Grant RCS20087Z006, the Fundamental Research Funds for the Central Universities under Grant 2010JBZ008, and the State Key Lab. of Rail Traffic Control and Safety (Contract No. RCS2010K008), Beijing Jiaotong University.

\section{References}

1. Briso-Rodriguez, C., Cruz, J. M., \& Alonso, J. I. (2007). Measurements and modeling of distributed antenna systems in railway tunnels. IEEE Transactions on Vehicular Technology, 56(5, Part 2), $2870-2879$.

2. Zhang, Y. P., \& Hwang, Y. (1997). Enhancement of rectangular tunnel waveguide model. Microwave Conference Proceedings 1997. APMC '97, 1997 Asia-Pacific, 1, 197-200.

3. Zhang, Y. P., \& Hwang, Y. (1998). Characterization of uhf radio propagation channels in tunnel environments for microcellular and personal communications. IEEE Transactions on Vehicular Technology, 47, 283-296.

4. Alonso, J., Izquierdo, B., \& Romeu, J. (2009). Break point analysis and modelling in subway tunnels. In 3rd European conference on ant. and propag (Vol. 49, pp. 3524-3258).

5. Guan, K., Zhong, Z. H. D., Ai, B., \& Briso-Rodriguez, C. (2010). Research of propagation characteristics of break point: Near zone and far zone under operational subway condition. In International conference on communications and mobile computing, communications and information theory symposium (pp. 114-118).

6. Zhang, Y. P. (2003). Novel model for propagation loss prediction in tunnels. IEEE Transactions on Vehicular Technology, 52, 1308-1314.

7. Molina-Garcia-Pardo, J. M., Lienard, M., Nasr, A., \& Degauque, P. (2008). On the possibility of interpreting field variations and polarization in arched tunnels using a model for propagation in rectangular or circular tunnels. IEEE Transactions on Antenna Propagation, 56(9), 1206-1211.

8. ETSI ETR 300-3 ed. 1 (2000-02): (2000). Terrestrial Trunked Radio (TETRA); Voice Plus Data (V+D); Designers' Guide; Part 3: Direct Mode Operation (DMO).

9. [Online]. Available: http://www.uic.asso.fr

10. IEEE Standard for Communications-Based Train Control (CBTC). (1999). Performance and functional requirements, 30 .

11. Notice of proposed rulemaking and order FCC 03-324, Federal Communications Commission. Febrary (2003).

12. Mouly, M., \& Pautet, M.B. (1992). The GSM system for mobile communications, Paliseau, France.

13. The wireless dictionary Gilb, J.P.K. IEEE standards wireless series (2005).

14. IEEE draft standard for information technology-Telecommunications and information exchange between systems-Local and Metropolitan networks-specific requirements-Part II: Wireless LAN Medium Access Control (MAC) and Physical Layer (PHY) specifications: IEEE 802.11 Wireless Network Management Amendment, March (2010).

15. IEEE standard for local and metropolitan area networks Part 16: Air interface for broadband wireless access systems amendment 1: Multiple relay specification (pp. c1-290) (2009).

16. Zhang, Y. P., Zheng, G. X., \& Sheng, J. H. (2001). Radio propagation at $900 \mathrm{MHz}$ in underground coal mines. IEEE Transactions on Antennas Propagation, 49, 757-762. 
17. Klemenschits, T., \& Bonek, E. (1994). Radio coverage of road tunnels at 900 and $1800 \mathrm{MHz}$ by discrete antennas. In 5th IEEE international symposium on PIMRC (Vol. 2, pp. 411-415)

18. Hrovat, A., Kandus, G., \& Javornik, T. (2010). Four-slope channel model for path loss prediction in tunnels at $400 \mathrm{MHz}$. IET Microwaves Antennas and Propagation, 4, 571-582.

19. Guan, K., Zhong, Z. D., Ai, B., \& Briso-Rodriguez, C. (2001). Propagation mechanism analysis before the break point inside tunnels. Accepted by IEEE 74th vehicular technology conference.

20. Mariage, P., Lienard, M., \& Degauque, P. (1994). Theoretical and experimental approach of the propagation of high frequency waves in road tunnels. IEEE Transactions on Antennas Propagation, 42, 75-81.

21. Saunders, S. (2005). Antennas and propagation for wireless communication systems. Chichester, England: Wiley.

\section{Author Biographies}
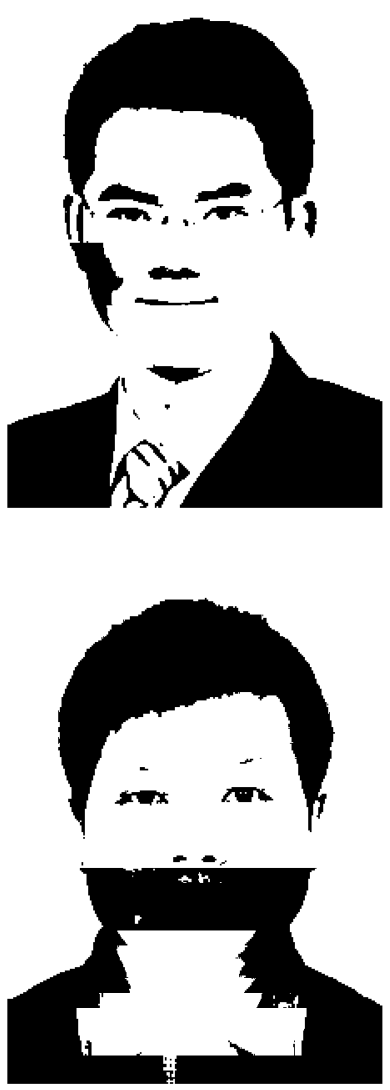

Ke Guan was born in Si Chuan, China, in 1983. He received the bachelor degree from Beijing Jiaotong University in 2006. From March, 2009 to August, 2009, he conducted research in Universidad Politécnica de Madrid, Madrid, Spain, as a visiting scientist. Since 2010, he is a Ph.D. candidate in the State Key Lab of Rail Traffic Control and Safety at Beijing Jiaotong University. His primary interest is in propagation and wireless channel measurements and modeling, especially under high speed condition.

Zhangdui Zhong was born in May 1962. He is now the professor and the director of doctor degree candidate in Beijing Jiaotong University. $\mathrm{He}$ is also the director of Modern Telecommunications Research Institute in Beijing Jiaotong University and the Chief Scientist of Ministry Railways of China. He has won lots of scientific and technical prizes in China and published over 90 scientific research papers. His interests are wireless network systems for railway, control theory and techniques for wireless railway, GSM-R system. 


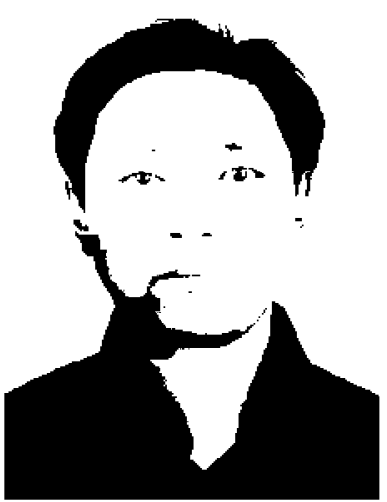

Bo Ai was born in Shaanxi Province in China in 1974. He received a B.Sc. Degree from Engineering College of Armed Police Force in 1997, a Master and Dr. degree from Xidian University in 2002 and 2004 in China, respectively. From 2005 to 2007, he worked as a Post Dr. research fellow in Dept. of E \& E, state key lab. on microwave and digital communications in Tsinghua University in China and graduated with great honors of Excellent Postdoctoral Research Fellow in Tsinghua University. He is now working in Beijing Jiaotong University as an associate professor. He is an editorial committee member of journal of "Wireless Personal Communications", "Recent Patents on Electrical Engineerin", "Computer Simulations", "Information and Electronic Engineering", an IEEE member and a senior member of Electronics Institute of China (CIE). He has published four books and 73 scientific papers in his research area till now. His bio. has been included in Marquis Who's Who in Science and Engineering and Cambridge IBC books. His current interests are the research and applications of OFDM techniques with emphasis on synchronization, HPA linearization techniques and GSM Railway systems.

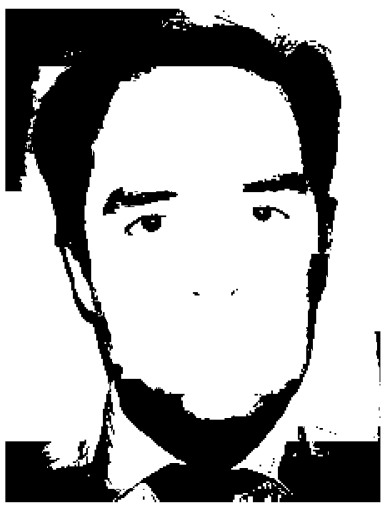

Cesar Briso-Rodríguez was born in Valladolid, Spain, in 1968. He received the Ingeniero de Telecomunicación and $\mathrm{Ph}$.D. degrees from the Universidad Politécnica de Madrid (UPM), Spain, in 1996 and 1999, respectively. Since 1996, he has been Full Professor of the EUIT Telecomunicación of the UPM. His research activity has been focused in the area of design and develop of high-frequency communications systems for complex environments. Prof. Briso-Rodríguez was awarded with the prize for the best Ph.D. in GSM communications by the Spanish association of Telecomunicacion engineers. 